\title{
Identification of the Relative Distribution of Rare-Earth Ions in Phosphate Glasses
}

\author{
Richard A. Martin, ${ }^{1}$ Philip S. Salmon, ${ }^{1, *}$ Henry E. Fischer, ${ }^{2}$ and Gabriel J. Cuello ${ }^{3}$ \\ ${ }^{1}$ Department of Physics, University of Bath, Bath BA2 7AY, United Kingdom \\ ${ }^{2}$ LURE, Centre Universitaire Paris-Sud, BP 34, F-91898, Orsay Cédex, France \\ ${ }^{3}$ Institut Laue-Langevin, BP 156, F-38042, Grenoble Cédex 9, France
}

(Received 23 January 2003; published 6 May 2003)

\begin{abstract}
The relative distribution of rare-earth ions $R^{3+}\left(\mathrm{Dy}^{3+}\right.$ or $\left.\mathrm{Ho}^{3+}\right)$ in the phosphate glass $R \mathrm{Al}_{0.30} \mathrm{P}_{3.05} \mathrm{O}_{9.62}$ was measured by employing the method of isomorphic substitution in neutron diffraction. It is found that 7.9(7) $R-R$ nearest neighbors reside at 5.62(6) $\AA$ in a network made from interlinked $\mathrm{PO}_{4}$ tetrahedra. Provided that the role of $\mathrm{Al}$ is explicitly considered, a self-consistent account of the local matrix atom correlations can be developed in which there are 1.68(9) bridging and 2.32(9) terminal oxygen atoms per phosphorus.
\end{abstract}

DOI: 10.1103/PhysRevLett.90.185501

Oxide glasses containing rare-earth ions, $R^{3+}$, have many fascinating opto-electronic and magneto-optical properties and find application as, e.g., lasers and Faraday rotators [1]. It is therefore desirable to understand the interactions between the rare-earth ions, and their mediation by the matrix material, in order to develop realistic microscopic models. Hence progress is dependent on the provision of unambiguous experimental information about both the relative distribution of the rare-earth ions and the structure of the glassy matrix. The literature on the $R-R$ distribution in oxide glasses is, however, notable by its paucity, which can be attributed largely to the structural complexity of topologically disordered materials.

In this Letter, we tackle the problem by applying the method of isomorphic substitution in neutron diffraction to measure the $R-R$ partial structure factor and related difference functions for the glassy phosphate $R \mathrm{Al}_{0.30} \mathrm{P}_{3.05} \mathrm{O}_{9.62}$, where $R$ denotes Dy or Ho. The choice of material was motivated by a general interest in rareearth phosphate glasses [2-4] and by a lack of success in identifying the nearest-neighbor $R$ - $R$ correlations, despite many recent attempts using a variety of techniques including neutron diffraction [5], x-ray diffraction [6,7], combinations of these methods $[8,9]$, and extended $x$-ray absorption fine structure spectroscopy [4,10]. From a practical point of view, a large mol \% of rare-earth ions may be incorporated into glassy phosphates, which enhances the prospect of identifying the $R-R$ correlations, and the inclusion of Al results in favorable mechanical and chemical properties; e.g., fibers may be drawn and the glasses are water resistant $[5,6,11]$. The structures of the crystalline metaphosphates, $c-R \mathrm{P}_{3} \mathrm{O}_{9}[12,13]$, and ultraphosphates, $c-R \mathrm{P}_{5} \mathrm{O}_{14}[14,15]$, comprising small rareearth cations are known and offer a guide for interpreting the neutron diffraction results.

At the heart of the isomorphic substitution method, which has recently been used with success to study molten rare-earth compounds $[16,17]$, is the assumption that glassy $R \mathrm{Al}_{0.30} \mathrm{P}_{3.05} \mathrm{O}_{9.62}$ samples can be made that are structurally the same and differ only in the coherent
PACS numbers: 61.43.Fs, 42.70.-a, 61.12.Ld, 81.05.Kf

neutron scattering length, $b_{R}$, of the rare-earth ion. Then, by making neutron diffraction experiments on three samples having sufficiently contrasting $b_{R}$ values, the $R-R$ partial structure factor, $S_{R R}(k)$, can be measured which enables a considerable reduction in the complexity of correlations associated with the total structure factor, $F(k)$, measured in a single diffraction experiment, where $k$ is the magnitude of the scattering vector [18-20]. Specifically, $F(k)$ can be separated into contributions from $S_{R R}(k)$ and from difference functions that involve only the $R$-matrix atom correlations, $\Delta_{R \mu}(k)$, or the matrixmatrix atom correlations, $\Delta_{\mu \mu^{\prime}}(k)$, where $\mu$ (or $\mu^{\prime}$ ) denotes $\mathrm{Al}, \mathrm{P}$, or $\mathrm{O}$. The latter are given by

$$
\begin{gathered}
\Delta_{R \mu}(k)=\sum_{\mu} 2 c_{R} c_{\mu} b_{\mu} \delta_{R}\left[S_{R \mu}(k)-1\right], \\
\Delta_{\mu \mu^{\prime}}(k)=\sum_{\mu} \sum_{\mu^{\prime}} c_{\mu} c_{\mu^{\prime}} b_{\mu} b_{\mu^{\prime}}\left[S_{\mu \mu^{\prime}}(k)-1\right],
\end{gathered}
$$

where $c_{\alpha}$ and $b_{\alpha}$ are the atomic fraction and coherent scattering length of chemical species $\alpha$, respectively, $\delta_{R}$ is a difference between the scattering lengths of the $R^{3+}$ ions, and $S_{\alpha \beta}(k)$ is a partial structure factor. The real-space functions corresponding to $S_{R R}(k), \Delta_{R \mu}(k)$, and $\Delta_{\mu \mu^{\prime}}(k)$ are the partial pair distribution function $g_{R R}(r)$ and the difference functions $\Delta G_{R \mu}(r)$ and $\Delta G_{\mu \mu^{\prime}}(r)$, respectively. The latter are obtained from Eqs. (1) and (2) by replacing the $S_{\alpha \beta}(k)$ by the corresponding partial pair distribution functions $g_{\alpha \beta}(r)$, and the limiting values $\Delta G_{R \mu}(0)$ and $\Delta G_{\mu \mu^{\prime}}(0)$ follow from setting $g_{\alpha \beta}(0)=0$. The mean coordination number of $\beta$ around $\alpha$ is denoted by $\bar{n}_{\alpha}^{\beta}$.

The three samples required for the diffraction experiments were made by fusing $\mathrm{Dy}_{2} \mathrm{O}_{3}(99.9 \%), \mathrm{Ho}_{2} \mathrm{O}_{3}$ (99.9\%), or an equal mixture of $\mathrm{Dy}_{2} \mathrm{O}_{3}$ and $\mathrm{Ho}_{2} \mathrm{O}_{3}$ with $\mathrm{P}_{2} \mathrm{O}_{5}(99 \%)$ in alumina $\left(\mathrm{Al}_{2} \mathrm{O}_{3}\right)$ crucibles. $\mathrm{Dy}^{3+}$ and $\mathrm{Ho}^{3+}$ were chosen as isomorphic pairs as they are adjacent in the periodic table, have comparable ionic radii $(0.912$ and $0.901 \AA$ ) [21], and Pettifor chemical parameters (0.685 
and 0.6825) [22], share a similar structural chemistry [23], and have contrasting coherent neutron scattering lengths [16.0(2) and 8.01(8) fm] [24]. The oxide powder mixtures were initially allowed to absorb a fixed small amount of atmospheric water at room temperature before the crucible was placed into a preheated oven at $500{ }^{\circ} \mathrm{C}$ for $1 \mathrm{~h}$. The crucible was then moved to another oven at $1000^{\circ} \mathrm{C}$, left for $30 \mathrm{~min}$, and finally transferred to a third oven at $1680^{\circ} \mathrm{C}$. After $30 \mathrm{~min}$ the melt was poured onto a graphite mold and annealed at $500{ }^{\circ} \mathrm{C}$ for $24 \mathrm{~h}$. Although an identical preparation method was used for all of the samples, the process by which the $\mathrm{Al}$ is incorporated into the glassy matrix is difficult to control, hence precluding the use of expensive rare-earth isotopes and application of the isotopic substitution method in neutron diffraction [18]. It was therefore necessary to prepare many samples and then select those with matching compositions, $1 R: 0.30(4) \mathrm{Al}: 3.05(11) \mathrm{P}: 9.62(35) \mathrm{O}$, after investigation using electron probe microanalysis. The glasses were transparent and homogeneous, and their atomic number density was $0.0715(6) \AA^{-3}$.

The diffraction experiments were made using the D4C instrument (Institut Laue-Langevin, Grenoble) with an incident neutron wavelength of $0.7100 \AA$. The paramagnetic differential scattering cross sections of $\mathrm{Dy}^{3+}$ and $\mathrm{Ho}^{3+}$ were evaluated using the method outlined elsewhere [16]. At each stage of the data analysis procedure, described in Ref. [19], a full set of reliability checks was performed to test the efficacy of the isomorphic assumption. For example, each measured difference function should obey the usual sum-rule relation and give rise to a well-behaved real-space function: this should oscillate about the calculated low- $r$ limit and, when the oscillations are set to this limit, its back Fourier transform should be in good overall agreement with the original reciprocal space data set. Furthermore, as three different routes may be used to construct the experimental difference functions [19], it was verified that the three $\Delta_{\mu \mu^{\prime}}(k)$ are the same within the statistical uncertainties and, likewise, the three $\Delta_{R \mu}(k) / \delta_{R}$. In the following, the $R-\mathrm{Al}$, $R$-P, and $R$-O weighting factors in Eq. (1) are $0.85(2)$, 12.8(3), and 45.7(1.2) mb while the Al-P, Al-O, Al-Al, $\mathrm{P}-\mathrm{O}, \mathrm{P}-\mathrm{P}$, and O-O weighting factors in Eq. (2) are 1.66(3), 5.92(6), 0.055(1), 89.4(1.2), 12.5(3), and 159.8(1.4) mb, respectively.

The dominant features in the measured partial structure factor $S_{R R}(k)$ [Fig. 1(a)] are two peaks at low $k$. This motivated a smooth truncation of the data, by application of a cosine window function over the region $4 \leq$ $k\left(\AA^{-1}\right) \leq 5$, prior to Fourier transformation in order to identify the corresponding main features in real space. The result, which is represented by the pair-correlation function $d_{R R}(r) \equiv 4 \pi n_{0} r\left[g_{R R}(r)-1\right]$ in Fig. 2(a), is a first peak at 5.65(2) $\AA$ giving $\bar{n}_{R}^{R}=8.6(2)$ with no clearly identifiable features at lower $r$. Next, the entire $k$-space range of $S_{R R}(k)$ was transformed, and the resultant $d_{R R}(r)$

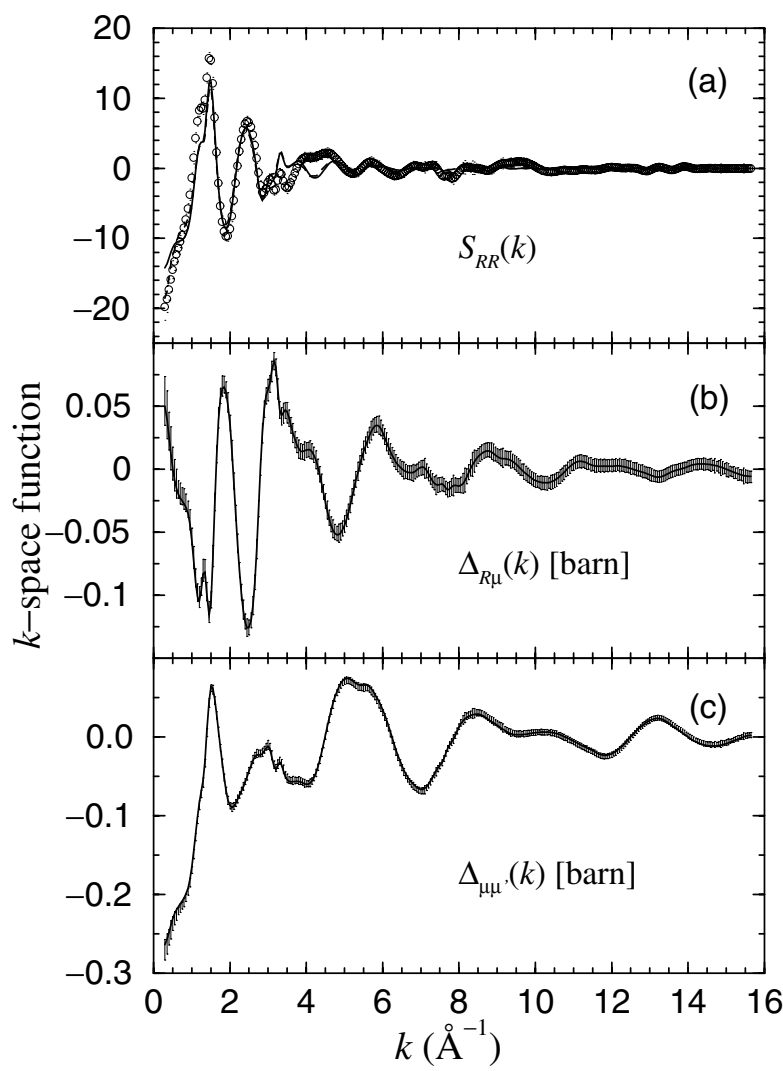

FIG. 1. The reciprocal space functions (a) $S_{R R}(k)$, (b) $\Delta_{R \mu}(k)$, and (c) $\Delta_{\mu \mu^{\prime}}(k)$ for glassy $R \mathrm{Al}_{0.30} \mathrm{P}_{3.05} \mathrm{O}_{9.62}$. The measured data points are represented either by circles (a) or by points with error bars, (b) and (c). The dashed curve in (a) gives the minimum information solution while the solid curves in (a), (b), and (c) give the back Fourier transforms of $d_{R R}(r), \Delta D_{R \mu}(r)$, or $\Delta D_{\mu \mu^{\prime}}(r)$ after the unphysical low- $r$ oscillations are set to the calculated limit of $-4 \pi n_{0} r$.

also shows a strong peak at a comparable position of 5.55(2) $\AA$ giving $\bar{n}_{R}^{R}=7.9(2)$. More pronounced features are observed at lower $r$ but these could not be definitively identified with unwanted correlations (cf. Ref. [20]); for example, when the oscillations for $0 \leq r(\AA) \leq 5.1$ (including the peak at $4.72 \AA$ ) are set to the low- $r$ limit of $-4 \pi n_{0} r$ and the data are back Fourier transformed, the resultant function is in reasonable agreement with the measured $S_{R R}(k)$ over the entire $k$-space range [Fig. 1(a)]. Finally, the minimum noise reconstruction method was used [25] which gives, essentially, a smooth $d_{R R}(r)$ that is constrained to take physical values, i.e., $d_{R R}(r)=$ $-4 \pi n_{0} r$ for $0 \leq r \leq r_{\min }$ and $d_{R R}(r) \geq-4 \pi n_{0} r$ for $r>$ $r_{\text {min }}$, with $r_{\text {min }}$ varied between 4.5 and $5.1 \AA$. A first peak at 5.65(2) A was obtained, with $\bar{n}_{R}^{R}=7.2(2)$, and no physical features could be generated for $4.5 \leq r(\AA) \leq$ 5.1. Thus, the diffraction data identify a first nearestneighbor $R-R$ distance of 5.62(6) $\AA$ with $\bar{n}_{R}^{R}=7.9(7)$ together with intermediate range ordering of the $R-R$ correlations extending beyond $15 \AA$ [Fig. 2(a)]. This picture is consistent with the structure of $c-R \mathrm{P}_{3} \mathrm{O}_{9}$, in 


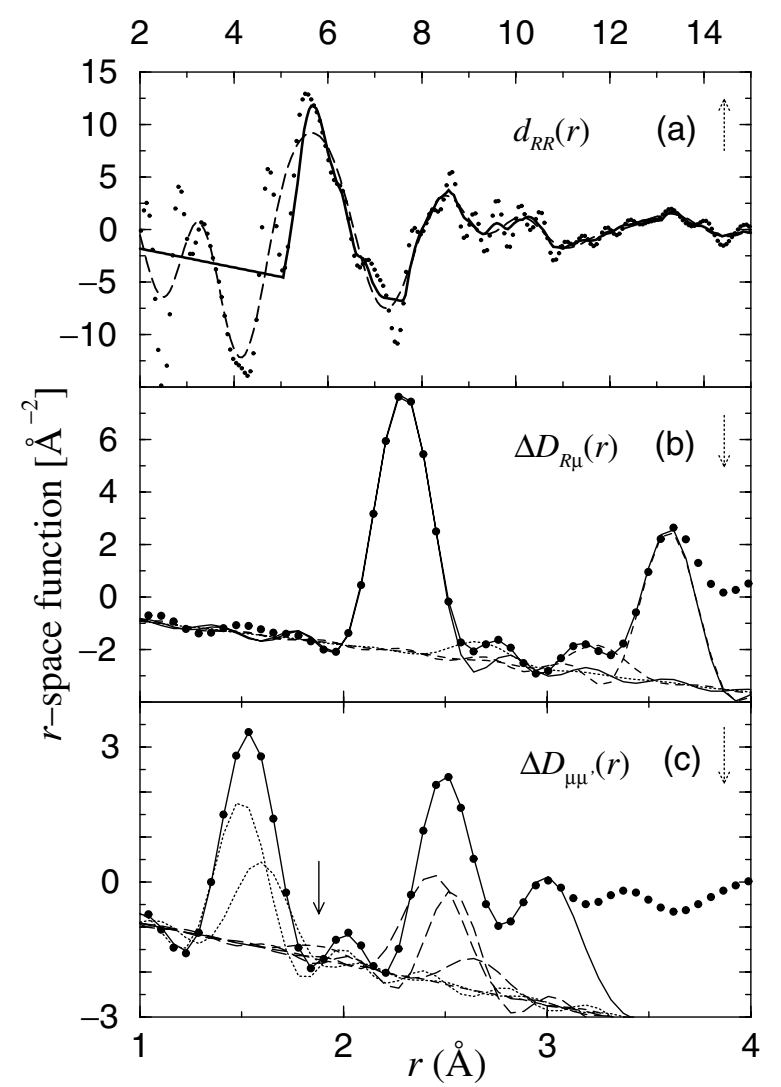

FIG. 2. The circles give the real-space functions (a) $d_{R R}(r)$, (b) $\Delta D_{R \mu}(r)$, and (c) $\Delta D_{\mu \mu^{\prime}}(r)$ obtained by Fourier transforming the reciprocal space functions of Fig. 1, and the dotted arrows point to the relevant abscissa scale. In (a) the solid curve gives the minimum information solution and the dashed curve shows the effect of smoothly truncating $S_{R R}(k)$ by using a cosine function. In (b) and (c), the solid curves give the fitted function and the other curves the individual convoluted Gaussians: in (b) $R$-O (dotted curves), $R$-P (dashed curves); in (c) P-O (dotted curves), Al-O (dashed curve), O-O (long dashed curves). Several of the larger- $r$ Gaussians are omitted from (c) for clarity of presentation. The solid arrow in (c) points to the Al-O Gaussian peak position.

which eight nearest-neighbor $R-R$ ions reside in the range 5.4-7.0 $\AA$ [13], and the absence of $R$ - $R$ correlations at $r \leq$ $4 \AA$ is in accord with other studies $[5,6,10]$.

The difference functions $\Delta_{R \mu}(k)$ and $\Delta_{\mu \mu^{\prime}}(k)$, shown in Figs. 1(b) and 1(c), have high- $k$ oscillations that are truncated by the finite measurement window function of the diffractometer $M\left(k \leq 15.65 \AA^{-1}\right)=1$, $M\left(k>15.65 \AA^{-1}\right)=0$. This effect was taken into explicit account by considering the corresponding $r$-space functions in the form

$$
\begin{gathered}
\Delta D_{R \mu}(r)=\frac{4 \pi n_{0} r}{\left|\Delta G_{R \mu}(0)\right|} \Delta G_{R \mu}(r) \otimes M(r), \\
\Delta D_{\mu \mu^{\prime}}(r)=\frac{4 \pi n_{0} r}{\left|\Delta G_{\mu \mu^{\prime}}(0)\right|} \Delta G_{\mu \mu^{\prime}}(r) \otimes M(r),
\end{gathered}
$$

where $\otimes$ denotes the one-dimensional convolution operator and $M(r)$, the Fourier transform of $M(k)$, takes the form of a symmetrical function. To enable those features that are an artifact of $M(r)$ to be distinguished, the first few peaks in $\Delta D_{R \mu}(r)$ or $\Delta D_{\mu \mu^{\prime}}(r)$ were fitted by least squares to a sum of Gaussians representing the partial pair-correlation functions convoluted with $M(r)$ and the results are shown in Figs. 2(b) and 2(c). In general, the peaks fitted at the larger- $r$ values are not expected to yield accurate parameters, owing to the overlap from correlations at even larger $r$, but were included to increase the reliability of the parameters that are reported for the peaks fitted at smaller $r$.

By comparison with the structures of $c-R \mathrm{P}_{3} \mathrm{O}_{9}$ and $c-R \mathrm{P}_{5} \mathrm{O}_{14}[12-15]$, the first peak in $\Delta D_{R \mu}(r)$ at $2.27 \AA$ is identified with $R-\mathrm{O}$ correlations and the second peak at $3.62 \AA$ with $R$-P correlations. The fit over the range $2.0 \leq$ $r(\AA) \leq 3.4$ gave a goodness-of-fit parameter $R_{\chi}$ [26] of $1.3 \%$ with Gaussians corresponding to $\bar{n}_{R}^{\mathrm{O}}=6.2(1)$ at 2.30(1) $\AA, \bar{n}_{R}^{\mathrm{O}}=0.5(1)$ at $2.67(1) \AA, \bar{n}_{R}^{\mathrm{P}}=1.1(1)$ at 3.25(2) $\AA$, and $\bar{n}_{R}^{\mathrm{P}}=6.0(1)$ at 3.60(2) $\AA$. The $R^{3+}$ ion coordination environment is therefore distorted relative to $c-R \mathrm{P}_{3} \mathrm{O}_{9}$, in which six $\mathrm{R}-\mathrm{O}$ neighbors reside in the range $2.17 \leq r(\AA) \leq 2.29$ [13], and the overall coordination number $\bar{n}_{R}^{\mathrm{O}}=6.7(1)$ in the glass compares with $\bar{n}_{R}^{\mathrm{O}}=8$ for $2.29 \leq r(\AA) \leq 2.41$ in $c-R \mathrm{P}_{5} \mathrm{O}_{14}$ [14].

The structure of crystalline rare-earth phosphates is based on networks whose connectivity is determined by the number of bridging, $\mathrm{O}_{\mathrm{B}}$, and terminal, $\mathrm{O}_{\mathrm{T}}$, oxygen atoms associated with the $\mathrm{PO}_{4}$ tetrahedral building blocks [12-15]. The first peak in $\Delta D_{\mu \mu^{\prime}}(r)$ at $1.53 \AA$ is attributed to the P-O correlations from these tetrahedra and the corresponding oxygen-oxygen correlations, denoted by $\mathrm{O}-(\mathrm{P})-\mathrm{O}$, will give a strong contribution to the second peak at $2.52 \AA$. The region between these two peaks, $1.7 \leq r(\AA) \leq 1.9$, will have a contribution from Al-O correlations since ${ }^{27} \mathrm{Al}$ nuclear magnetic resonance experiments, made on rare-earth phosphate glasses prepared in alumina crucibles [5], show that Al can be fourfold, fivefold, or sixfold coordinated to oxygen. In $c-\mathrm{AlP}_{3} \mathrm{O}_{9}$ [27], aluminum is octahedrally coordinated to oxygen at a distance $r_{\mathrm{AlO}}=1.88 \AA$ giving an $\mathrm{O}-(\mathrm{Al})-\mathrm{O}$ nearest-neighbor separation of $\sqrt{2} r_{\mathrm{AlO}}=2.66 \AA$, placing these correlations under the second peak in $\Delta D_{\mu \mu^{\prime}}(r)$. By comparison, for tetrahedral coordination $r_{\mathrm{AlO}}=1.76 \AA$ [28] giving an $\mathrm{O}-(\mathrm{Al})-\mathrm{O}$ distance of $\sqrt{8 / 3} r_{\mathrm{AlO}}=2.87 \AA$ in the region between the second and third peaks in $\Delta D_{\mu \mu^{\prime}}(r)$. The second peak has, therefore, contributions only from $\mathrm{O}-(\mathrm{P})-\mathrm{O}$ and $\mathrm{O}-(\mathrm{Al})-\mathrm{O}$ correlations while the third peak was modeled assuming $\mathrm{P}-(\mathrm{OP})-\mathrm{O}, \mathrm{P}-(\mathrm{O})-\mathrm{P}$, $\mathrm{O}-(R)-\mathrm{O}$, and $\mathrm{Al}-(\mathrm{O})-\mathrm{P}$ correlations using the structures of $c-R \mathrm{P}_{3} \mathrm{O}_{9}[12,13]$ and $c-\mathrm{AlP}_{3} \mathrm{O}_{9}$ [27] as starting points.

The fit to $\Delta D_{\mu \mu^{\prime}}(r)$ over the range $1.2 \leq r(\AA) \leq 2.9$ gave $R_{\chi}=2.0 \%$ [see Fig. 2(c)]. Two Gaussians were used to represent the first peak, with $\bar{n}_{\mathrm{P}}^{\mathrm{O}_{\mathrm{T}}}=2.32(9)$ at $1.50(1) \AA$ and $\bar{n}_{\mathrm{P}} \mathrm{O}_{\mathrm{B}}=1.68(9)$ at $1.60(1) \AA$, giving an overall $\mathrm{P}-\mathrm{O}$ 
coordination number $\bar{n}_{\mathrm{P}}^{\mathrm{O}}=4.0(1)$. A distance $r_{\mathrm{AlO}}=$ 1.89(1) $\AA$ was found, in accord with Refs. [5,6], with $\bar{n}_{\mathrm{Al}}^{\mathrm{O}}=5.5(5)$. The second peak was fitted with Gaussians centered at 2.45(1), 2.54(1), and 2.65(1) A corresponding to $\mathrm{O}-(\mathrm{P})-\mathrm{O}$ coordination numbers of 2.3(1) and 1.4(1) and an $\mathrm{O}-(\mathrm{Al})-\mathrm{O}$ coordination number of $0.7(1)$, respectively, i.e., the overall $\mathrm{O}-(\mathrm{P})-\mathrm{O}$ nearestneighbor coordination number $\bar{n}_{\mathrm{O}}^{\mathrm{O}}=3.7(1)$. The small peak at $2 \AA$ was found to be mostly an artifact of $M(r)$, the effect of which may be reduced by using a diffractometer which accesses a larger- $k$ range.

In crystalline and glassy $\mathrm{P}_{2} \mathrm{O}_{5}$, a network is built from corner-sharing $\mathrm{PO}_{4}$ tetrahedra comprising three $\mathrm{O}_{\mathrm{B}}$ and one $\mathrm{O}_{\mathrm{T}}$ [3]. In the model of Hoppe and co-workers [29], the addition of a network modifier such as $R_{2} \mathrm{O}_{3}$ leaves the $\mathrm{PO}_{4}$ tetrahedra intact but depolymerizes the phosphate network through the breakage of $\mathrm{P}-\mathrm{O}_{\mathrm{B}}-\mathrm{P}$ bonds, thereby increasing the fraction of $\mathrm{O}_{\mathrm{T}}$ to which the $R^{3+}$ ions are exclusively bound via $\mathrm{P}-\mathrm{O}_{\mathrm{T}}-R$ linkages. Specifically, if $y$ is the number of oxygens added per $\mathrm{P}_{2} \mathrm{O}_{5}$ unit from $R_{2} \mathrm{O}_{3}$, the $\mathrm{P}: \mathrm{O}_{\mathrm{B}}: \mathrm{O}_{\mathrm{T}}$ ratio changes from $2: 3: 2$ in pure $\mathrm{P}_{2} \mathrm{O}_{5}$ to $2:(3-y): 2(1+y)$ in the modified material. The expressions $\bar{n}_{\mathrm{O}_{\mathrm{B}}}^{\mathrm{O}}=6(3-y) /(5+y)$ and $\bar{n}_{\mathrm{O}_{\mathrm{T}}}^{\mathrm{O}}=6(1+y) /$ $(5+y)$ then follow from taking oxygen-oxygen coordination numbers of 6 and 3 for the $\mathrm{O}_{\mathrm{B}}$ and $\mathrm{O}_{\mathrm{T}}$ sites, respectively, giving an overall $\mathrm{O}-(\mathrm{P})-\mathrm{O}$ nearest-neighbor coordination number $\bar{n}_{\mathrm{O}}^{\mathrm{O}}=24 /(5+y)$.

For glassy $R \mathrm{Al}_{0.30} \mathrm{P}_{3.05} \mathrm{O}_{9.62}$, the $\mathrm{Al}-\mathrm{O}$ coordination parameters suggest that a substantial number of the $\mathrm{Al}$ atoms adopt octahedral conformations as in crystalline [27] and glassy [30] $\mathrm{AlP}_{3} \mathrm{O}_{9}$-i.e., $\mathrm{Al}_{2} \mathrm{O}_{3}$ appears to act primarily as a network modifier, akin to $R_{2} \mathrm{O}_{3}$, from which we deduce $y=1.320$. Hence each $\mathrm{P}$ is on average bound to $1.68 \mathrm{O}_{\mathrm{B}}$ and $2.32 \mathrm{O}_{\mathrm{T}}$ with $\bar{n}_{\mathrm{O}_{\mathrm{B}}}^{\mathrm{O}}=1.59$ and $\bar{n}_{\mathrm{O}_{\mathrm{T}}}^{\mathrm{O}}=2.20$ giving an overall $\mathrm{O}-(\mathrm{P})-\mathrm{O}$ coordination number $\bar{n}_{\mathrm{O}}^{\mathrm{O}}=3.79$. Also, for sixfold coordinated $\mathrm{Al}$ an $\mathrm{O}_{\mathrm{T}}-(\mathrm{Al})-\mathrm{O}_{\mathrm{T}}$ coordination number of $\bar{n}_{\mathrm{O}}^{\mathrm{O}}=48 c_{\mathrm{Al}} /$ $(5+y) c_{\mathrm{P}}=0.75$ at $\sqrt{2} r_{\mathrm{AlO}}=2.66 \AA$ is anticipated. All of these parameters are in accord with those obtained from the fit to $\Delta D_{\mu \mu^{\prime}}(r)$ [Fig. 2(c)] which implies that the Hoppe et al. model [29] can act as an excellent starting point for understanding the structure of rare-earth phosphate glasses, even when they incorporate a significant mol $\%$ of $\mathrm{Al}$ impurity atoms. The predominant role of the latter is to bridge $\mathrm{PO}_{4}$ tetrahedra via $\mathrm{O}_{\mathrm{T}}-\mathrm{Al}-\mathrm{O}_{\mathrm{T}}$ linkages, thereby strengthening $[5,6]$ the glass network. For $\bar{n}_{R}^{\mathrm{O}}=$ 6.7 and $\bar{n}_{\mathrm{Al}}^{\mathrm{O}}=5.5$ an $18 \%$ sharing of the available $\mathrm{O}_{\mathrm{T}}$ between $R$ or Al centered coordination polyhedra can be deduced.

The present work demonstrates that it is possible to identify the $R-R$ correlations in phosphate glasses and provide a detailed examination of their matrix structure by using the method of isomorphic substitution in neutron diffraction. It therefore provides a new stimulus for the development of realistic microscopic models for these materials and the approach can be used as an alternative to the method of isotopic substitution in neutron diffraction to identify the relative distribution of rare-earth ions in a variety of vitreous oxides.

We gratefully thank Peter Taylor and Hugh Perrott for access to furnaces and for the EPMA analysis, and the EPSRC for financial support.

*Corresponding author.

[1] M. J. Weber, in Materials Science and Technology, edited by J. Zarzycki (VCH, Weinheim, 1991), Vol. 9, p. 619.

[2] C.-K. Loong et al., Physica (Amsterdam) 241-243B, 890 (1998).

[3] R. K. Brow, J. Non-Cryst. Solids 263-264, 1 (2000).

[4] M. Karabulut et al., Phys. Rev. B 65, 104206 (2002).

[5] J. M. Cole et al., J. Phys. Condens. Matter 11, 9165 (1999).

[6] J. M. Cole et al., J. Phys. Condens. Matter 13, 4105 (2001).

[7] U. Hoppe et al., J. Non-Cryst. Solids 297, 263 (2002).

[8] U. Hoppe et al., J. Non-Cryst. Solids 232-234, 44 (1998).

[9] U. Hoppe et al., Z. Naturforsch. 56a, 237 (2001).

[10] J. M. Cole et al., J. Phys. Condens. Matter 13, 6659 (2001).

[11] A. Mierzejewski et al., J. Non-Cryst. Solids 104, 323 (1988).

[12] H. Y-P. Hong, Acta Crystallogr. B 30, 1857 (1974).

[13] G. I. Dorokhova and O. G. Karpov, Sov. Phys. Crystallogr. 29, 400 (1984).

[14] M. Bagieu et al., Cryst. Struct. Commun. 3, 387 (1973).

[15] H. Y-P. Hong and J.W. Pierce, Mater. Res. Bull. 9, 179 (1974).

[16] J. C. Wasse and P. S. Salmon, J. Phys. Condens. Matter 11, 1381 (1999).

[17] J. C. Wasse et al., J. Phys. Condens. Matter 12, 9539 (2000).

[18] C. J. Benmore and P. S. Salmon, Phys. Rev. Lett. 73, 264 (1994).

[19] P. S. Salmon et al., Phys. Rev. B 58, 6115 (1998).

[20] P. S. Salmon and S. Xin, Phys. Rev. B 65, 064202 (2002).

[21] R. D. Shannon, Acta Crystallogr. A 32, 751 (1976).

[22] D. G. Pettifor, J. Phys. C 19, 285 (1986).

[23] A. F. Wells, Structural Inorganic Chemistry (Clarendon Press, Oxford, 1984), 5th ed.

[24] $b_{\alpha}(\alpha=\mathrm{Ho}, \mathrm{Al}, \mathrm{P}$, or O) was taken from V. F. Sears, Neutron News 3, 26 (1992); $b_{\text {Dy }}$ at the incident neutron energy of $162.3 \mathrm{meV}$ was calculated using the method of C. Cossy et al., J. Chem. Phys. 90, 3254 (1989) taking into account a resonance for ${ }^{164} \mathrm{Dy}$ at $-1.88 \mathrm{eV}$.

[25] A. K. Soper et al., Phys. Rev. E 47, 2598 (1993).

[26] D. I. Grimley et al., J. Non-Cryst. Solids 119, 49 (1990).

[27] H. van der Meer, Acta Crystallogr. B 32, 2423 (1976).

[28] A. C. Hannon and J. M. Parker, J. Non-Cryst. Solids 274, 102 (2000).

[29] U. Hoppe, J. Non-Cryst. Solids 195, 138 (1996).

[30] U. Hoppe et al., Z. Naturforsch. A 50, 684 (1995). 